
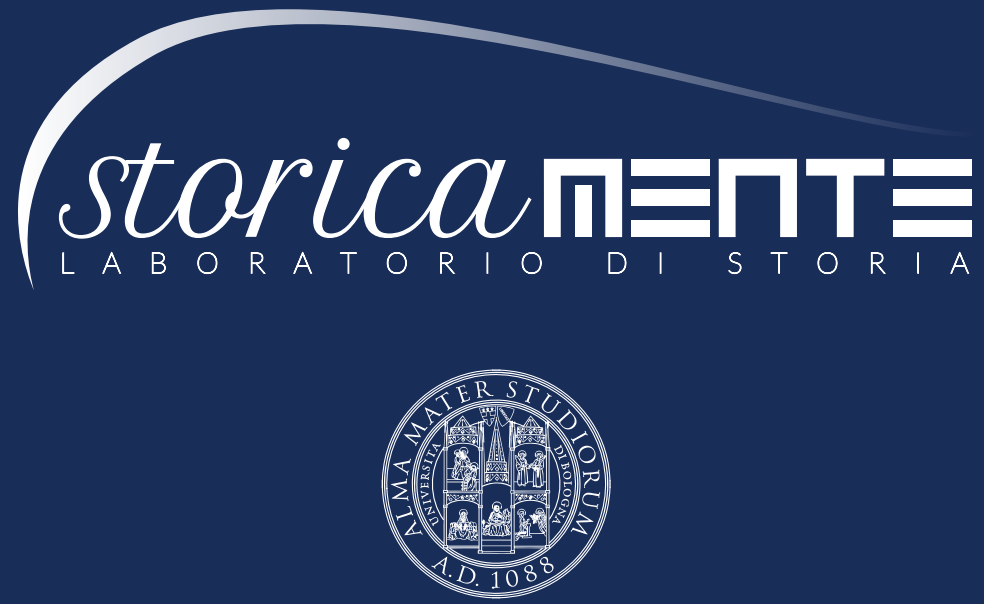

ALMA MATER STUDIORUM

Università di Bologna

Dipartimento di Storia Culture Civiltà

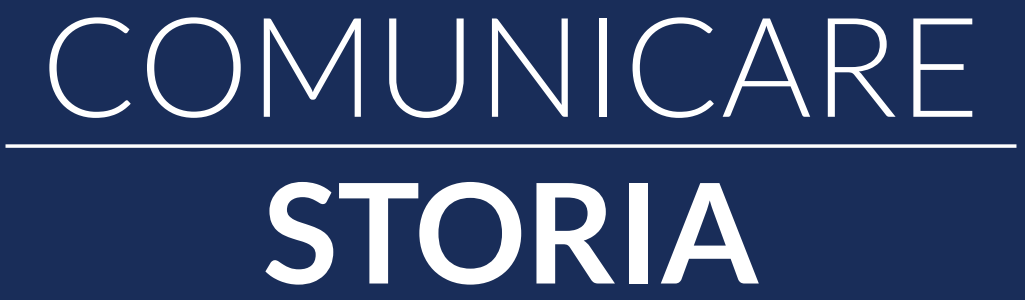

VIELLA 


\section{STORICAMENTE.ORG}

\section{Laboratorio di Storia}

\section{Francesco Pitassio}

Il cinema italiano del secondo dopoguerra e la controversa

questione della discontinuità: nuove strade, antichi territori?

(C) Francesco Pitassio

Creative Commons BY-NC-ND 4.0

International License 2004-2022

Volume: 2021

Issue: 24

Article number 15

Section: Comunicare storia

Pages. 1-28

DOI: $10.52056 / 9788833138732 / 24$

ISSN: $1825-411 \mathrm{X}$

Publisher: Viella

Double blind peer review: Yes

Document type: Editorial material

Research Areas: History

Published: 1/2/2022

Corresponding Address: Francesco Pitassio, Univ. Udine, Dipartimento di Studi Umanistici e del Patrimonio Culturale, Vicolo Florio 2/b, 33100, Udine, Italy 


\title{
Il cinema italiano del secondo dopoguerra e la controversa questione della discontinuità: nuove strade, antichi territori?
}

\author{
Francesco Pitassio \\ Univ. Udine, Dipartimento di Studi Umanistici e del Patrimonio Culturale \\ francesco.pitassio@uniud.it
}

Italian Cinema after the Second World War and the Controversial Question of Discontinuity: New Roads, Old Territories?

The article focuses on the Italian cinema in building collective memory in post-war transitional phase. It tackles neorealist humanism and descriptive style, together with its obliteration of Fascism. These features contribute to 'cultural trauma': a symbolic activity reshaping collective identity. Finally, the article scrutinizes non-fiction works representing a contested area, in political and historical terms: Trieste. This final pace sheds light on the degree of diffusion of cultural trauma and clarifies how the concealment of Fascism operated as parapraxis.

KEYWORDS: FILM STUDIES, CINEMA, ITALIAN NEOREALISM, DOCUMENTARY FILM, CULTURAL TRAUMA, TRIEST

\section{Mnemosyne. La mappa della memoria}

Per decenni, gli storici del cinema italiani e stranieri hanno considerato Paisà (Roberto Rossellini, 1946) come uno dei più puri esiti del cinema neorealista italiano. Nel discutere il film, il critico e teorico francese André Bazin, principale esegeta e alfiere di questa produzione, ne individuava le caratteristiche più rimarchevoli. Se ne ritengano qui alcune, particolarmente funzionali alla argomentazione che s'intende di seguito disporre. In prima istanza, la contemporaneità con la realtà che la narrazione del film descriveva. Nelle parole di Bazin, "in Italia Resistenza e Liberazione non sono affatto, come la rivolta di Parigi, semplici frasi storiche. Rossellini ha girato Paisà in un periodo in cui il suo racconto era ancora attuale" (Bazin 1986, 279). Si tratta di una "adesione spirituale all'epoca", da cui consegue una seconda qualità precipua del fe- 
nomeno cinematografico e culturale: l'adesione alla realtà descritta, al di qua di ogni identità ideologica, al cui centro è posto l'essere umano. "In un mondo ancora e già ossessionato dal terrore e dall'odio, in cui la realtà non è più quasi mai amata per se stessa ma solo rifiutata o difesa come segno politico, il cinema italiano è il solo a salvare, nel seno stesso dell'epoca che dipinge, un umanesimo rivoluzionario" (ivi, 280). Da terzo, l'immediatezza, originata in una sensibilità intuitiva, prossima all'oralità, anziché al disegno narrativo, e capace di cogliere nel modo più rispettoso quanto registrato:

Il cinema italiano possiede quell'andamento da reportage, quella naturalezza più vicina al racconto orale che alla scrittura, più allo schizzo che al dipinto. Ci voleva la spigliatezza e l'occhio sicuro di Rossellini, di Lattuada, di Vergano e di De Santis. La loro macchina da presa possiede un tatto cinematografico molto delicato, delle antenne meravigliosamente sensibili, che gli permettono di cogliere d'un tratto quel che si deve, come si deve (ivi, 293).

Da ultimo, la valenza autonoma di ogni singola immagine, equiparata a un fatto, di cui preserva la densità e unicità: le immagini non sono scelte ai fini di una tesi precostituita da una intenzionalità che le precede, ma ognuna è "frammento di realtà bruta, in se stesso multiplo ed equivoco, il cui 'senso' viene fuori solo a posteriori grazie ad altri 'fatti' tra i quali lo spirito stabilisce dei rapporti. Senza dubbio il regista ha ben scelto questi 'fatti', ma rispettando la loro integrità di 'fatto'” (ivi, 299).

Non mette qui conto esaminare o contestare la tenuta teorica o l'accuratezza storiografica di queste pagine di Bazin. Né evidenziare la capacità di canonizzare e cristallizzare un fenomeno multiforme e caotico, quale la produzione cinematografica dell'età neorealista, in una serie di caratteristiche distintive: contemporaneità, amore per la realtà e umanesimo, immediatezza, immagine-fatto. Piuttosto, si vuole qui esaminare in quale misura questi termini siano stati pietre angolari della cultura del neorealismo. Perché essa si fonda su un postulato, nel momento della sua genesi e affermazione: quanto segue la caduta del Fascismo non solo è in discontinuità con esso, ma è radicalmente e irriducibilmente differente da esso. Anzi, esso origina in una netta cesura, dalla quale i sopravvissuti alla catastrofe del totalitarismo italiano si erigono a giudici di quanto li ha preceduti. Nell'esporre il programma dell'Associazione Cultura- 


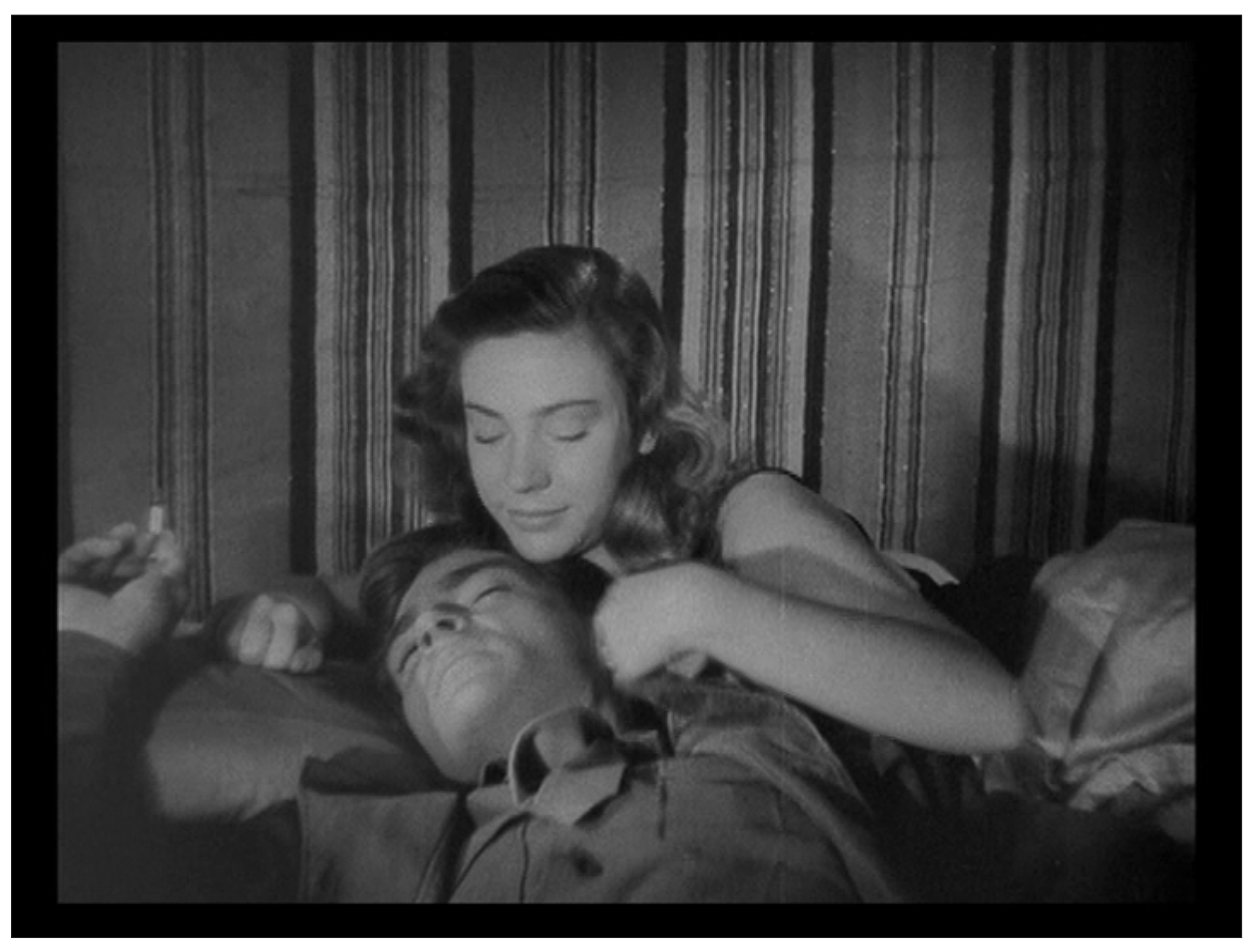

Fig. 1. Il terzo episodio di "Paisà" (R. Rossellini, 1946). Un ricordo dislocato.

le Cinematografica Italiana, alla fine del 1944, asseriva bellicosamente Cesare Zavattini: "La prima conferenza avrà un titolo processuale: 'Atto di accusa contro il cinema italiano'. Noi crediamo di sopravvivere, non solo, ma di crescere, in virtù di questa forza di ricerca intorno al passato che diventa per la sua radicalità garanzia dell'avvenire" (Zavattini 2002, 663). Ma se una cesura è così netta, se la presa di distanza così marcata, la possibilità reale di interrogarsi sul passato è altrettanto limitata: la cultura del neorealismo, la sua contemporaneità, immediatezza, umanesimo e realismo sono forse una soluzione per sviluppare un'etica senza rivolgere lo sguardo dove i nodi sono troppo stretti per essere districati. Quanto si vuole di seguito argomentare è che la cultura del neorealismo sia stata così efficace perché pienamente congruente con le esigenze di una complessa transizione sociale, politica e culturale.

$\mathrm{Si}$ proceda con ordine, muovendo da una incongruenza. Una svista per Bazin, una disomogeneità per il film diretto da Roberto Rossellini. Paisà, notoriamente, è costituito da una successione di episodi autonomi, inter- 
calati da sequenze con immagini documentarie di repertorio: gli uni e le altre seguono il movimento progressivo delle truppe alleate lungo la dorsale della penisola italiana, dalla Sicilia al Delta del Po. Apparentemente, la narrazione è lineare, sul piano temporale e spaziale, e questa linearità riprende una medesima consequenzialità storica. Tuttavia, il terzo episodio, posto al centro dell'opera, sul piano del tempo della narrazione, e su quello geografico e simbolico, dato che si svolge a Roma, palesa una contraddizione. Vi si narra dell'incontro in un locale tra Fred, un carrista statunitense (Gar Moore), e una ragazza romana (Maria Michi): lui molto ubriaco, lei pronta ad adescarlo, con l'implicita motivazione di procurarsi denaro o generi di necessità, in una capitale flagellata dalle ristrettezze belliche e dall'occupazione alleata. Giunti nella stanza della ragazza, il soldato rifiuta malamente le sue attenzioni e biascicando ricorda il giorno luminoso dell'ingresso delle truppe alleate a Roma, quando ebbe modo di conoscere una giovane borghese fresca e semplice, che lo accolse e gli offrì dell'acqua per ristorarsi, dopo la marcia nei carri armati. Dopo aver rimpianto quanto fosse diverso, allora, e le ragazze felici e sorridenti, Fred menziona Francesca, dando il via a un flashback. In esso, si ricostruiscono quelle circostanze: le immagini mostrano l'incontro tra il militare e una ragazza, la quale altri non è che la sua attuale adescatrice. Ma il soldato è troppo ebbro per collegare il proprio ricordo alla realtà attuale, e non riconosce nella seduttrice Francesca; la ragazza troppo soverchiata dalla vergogna per rivelarsi. Lei lo invita a recarsi all’indomani all'abitazione di Francesca, dove la troverà. Lui invece non darà seguito all'invito e, disprezzando coi commilitoni la derelitta che lo aveva sedotto la notte precedente, riprende la via del Nord. Cosa suggerisce questo episodio? In prima istanza, che la linearità e consequenzialità cronologica del neorealismo cinematografico non sono postulati indiscutibili, ma possono occasionalmente essere contraddette. Infatti, l'episodio romano oppone un tempo presente a un passato, convocato attraverso un'analessi. In secondo luogo, che questa variante viene spesso rigettata dagli osservatori, che "have gone to the extremes, describing it as 'Hollywood' filmmaking, using 'Hollywood' as a term of critical abuse [...]: commentators are pointing to a stylistic, narrative, and dramatic contrast between the Rome episode and the others in the film" (Wagstaff 2007, 196). Infine, che il ricordo non è affare degli italiani, ma compito di un osservatore esterno; questi, tuttavia, è perso nelle proprie nebbie e non riesce a uti- 
lizzare la propria memoria per comprendere la realtà in cui è immerso e le proprie responsabilità nel determinarla; alla stessa stregua, la ragazza, che esemplifica la realtà trasformata così profondamente dalla presenza delle truppe alleate, non può che subire questo cambiamento e soggiacere a una rappresentazione che prima e dopo l'ha dipinta in base a schemi incapaci di coglierne l'unicità. Ella non può che testimoniare la propria passione e passività. Le immagini dell'episodio romano di Paisà non sono contemporanee, prendono posizione rispetto alle contraddizioni antropologiche e geopolitiche, non paiono esito di una immediatezza rappresentativa - anzi, sul piano stilistico sono le immagini più ricercate di un'opera altrimenti scabra -, ubbidiscono a un disegno temporale e narrativo superiore. E dispiegano queste caratteristiche in un racconto in cui il problema del ricordo è centrale. Come mai? Che ruolo riveste una simile incoerenza? Vi si farà ritorno.

La produzione cinematografica neorealista è stata spesso indicata come immemore. Nelle parole di Giuliana Minghelli, la forza e rilevanza delle immagini di questa produzione "stems from conflicting imperatives: the commitment to bear witness and an interdiction against remembrance" (Minghelli 2012, 3). Prima di lei, Gian Piero Brunetta aveva discusso della produzione postbellica come caratterizzata da una profonda amnesia e da un patto del silenzio (Brunetta 1996). Ma testimonianza di cosa? E ricordo di cosa? Il punto di partenza delle riflessioni che tentano di rispondere a questi quesiti è l'approccio strutturalista alla memoria collettiva, originato negli studi di Maurice Halbwachs (Halbwachs 1987; 1997), ripreso e sistematizzato nel lavoro del sociologo israeliano Eviatar Zerubavel (Zerubavel 2005). Se lo studioso francese tra anni Venti e Trenta postulava l'esistenza di quadri della memoria, che determinano delle comunità di memoria e offrono la griglia su cui disporre i ricordi individuali, il secondo evidenzia la rilevanza delle relazioni reciproche tra i componenti di un quadro della memoria: essi traggono il proprio senso da questi rapporti: "Io credo che il significato storico degli eventi stia nel modo in cui essi sono situati nella nostra mente rispetto ad altri eventi. E infatti è la loro posizione strutturale in seno a tali scenari storici [...] che ci fa ricordare gli eventi del passato proprio come facciamo" (ivi, 28). Ora, le narrazioni del cinema neorealista del secondo dopoguerra hanno un termine a quo quasi sempre posto nell'armistizio dell' 8 settembre 1943: questa origine cronologi- 


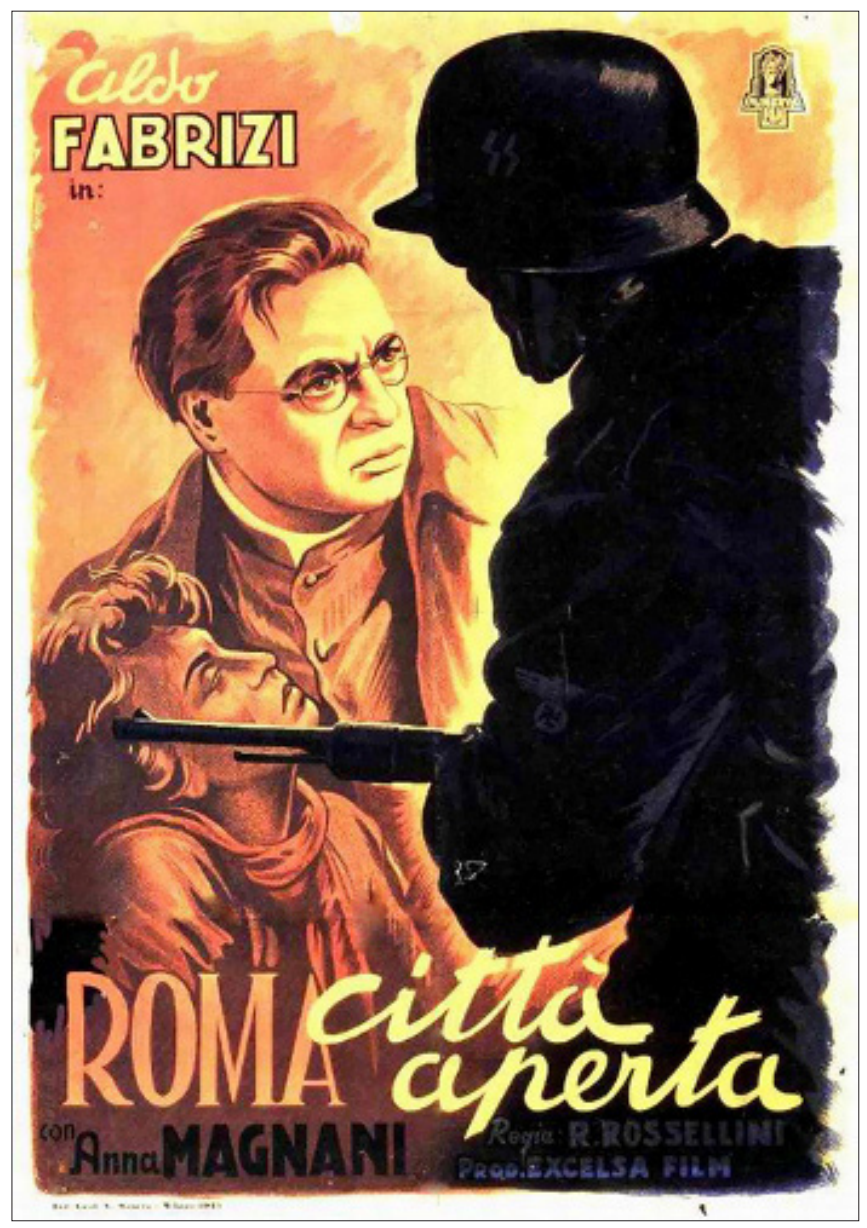

Fig. 2. Il manifesto di "Roma, città aperta" ( $R$. Rossellini, 1945). Il cattivo tedesco.

ca consente di dispiegare regolarmente delle narrazioni che sono di caduta - l'armistizio come onta nazionale - e ascesa - la Resistenza e le contraddizioni postbelliche come occasioni di riscatto o trasformazione del senso di cittadinanza e identità. Che si tratti della resistenza urbana attiva o passiva dei protagonisti di Roma, città aperta (Roberto Rossellini, 1945), della scoperta della militanza per i personaggi di Il sole sorge ancora (Aldo Vergano, 1946) o della esigenza di ricostituire i legami comunitari e siglare un patto di classe in Caccia tragica (Giuseppe De Santis, 1947), il racconto identifica sempre il punto più basso della comunità nazionale nella occupazione tedesca, per tracciare poi un percorso più o meno riuscito di redenzione.

L'altro assunto da cui muovere è l'affermazione di una nuova forma di memoria, sortita dal secondo conflitto mondiale. Il celebre aforisma di 
Adorno, secondo il quale "scrivere una poesia dopo Auschwitz è un atto di barbarie" (Adorno 1949, 22), ha meno a che vedere con l'attività lirica di per sé e assai di più con l'impotenza delle grandi narrazioni di dare il senso di un progresso umano e, dunque, di una teleologia. Quanto emerge dal disastro della Shoah e della guerra mondiale è una differente temporalità, in una tradizione speculativa all'origine di una branca dei trauma studies: "Just as modern science ushered in an era of progressive temporality, the Holocaust is thus seen as having ushered in 'traumatic temporality', the hallmark of which is supposedly an inability to tell a linear narrative" (Olick, Vinitzky-Seroussi e Levy 2011, 30-1). Si tratta, in prima istanza, di un differente ritmo indotto dal conflitto mondiale e dalle sue conseguenze, dato che uomini e donne "were torn by war from one set of daily rhythms and were in search of another" (Winter e Sivan 1999, 10). Ora, questa condizione di sospensione è particolarmente rilevante, ad avviso di chi scrive. Nello scenario postbellico la Shoah ha una valenza esemplificativa, nella sua eccezionalità, di un processo lacerante per numerose comunità di memoria, particolarmente quelle europee. Nel secondo dopoguerra esse adottano un "oblio dialogico" (Assmann 2011), ovvero un patto del silenzio, funzionale a ricostituire i legami sociali in conseguenza di un conflitto generalizzato, spesso civile. Dunque, l'enormità della Seconda guerra mondiale e dei suoi aspetti più atroci, unitamente alle lacerazioni delle comunità nazionali, induce nuove tipologie di memoria.

Si ritiene che il neorealismo cinematografico italiano risponda in modo particolarmente appropriato alle esigenze sociali, culturali e politiche italiane e internazionali di questo nuovo modo di ricordare e dimenticare. La produzione cinematografica neorealista, infatti, imperniandosi sulla rappresentazione dimessa dell'esperienza resistenziale, come in Paisà, Roma, città aperta, Il sole sorge ancora, ma anche nei meno celebrati Due lettere anonime (Mario Camerini, 1945) e Un giorno nella vita (Alessandro Blasetti, 1946), o descrivendo le difficoltà postbelliche, alla stregua di Sciuscià (Vittorio De Sica, 1946), Ladri di biciclette (Vittorio De Sica, 1948) o Riso amaro (Giuseppe De Santis, 1948), sembra rifuggire i rischi di una prospettiva univoca, ma anche di ricordare troppo. Nel partecipare di questa cultura, il cinema del neorealismo italiano, tuttavia, realizza quello che il teorico e storico del cinema anglo-germanico Thomas Elsaesser ha definito poetica della paraprassia (atto mancato) 
(Elsaesser 2014). Nel riprendere il termine dalla riflessione di Freud, Elsaesser appunta la propria attenzione su un aspetto: il concetto di atto mancato implica due componenti, uno difettoso (fehlen) e l'altro performativo (leisten); dunque, l'atto mancato non è semplicemente l'inconcludenza di un'azione, ma l'esibizione di questa lacuna. Ora, la costante esclusione del passato nazionale dalla rappresentazione si configura come valorizzazione della latitanza: da un lato, i film del neorealismo e, segnatamente, i suoi capolavori contribuiscono a forgiare la narrazione di una rinascita, dato che "for many Italians, in fact, the end of the war seemed to signal a new life for the nation" (Leavitt 2016, 10); dall'altro, indicano platealmente un'assenza, presente tuttavia nella esperienza pubblica e privata dell'intera nazione. Sul piano storico, si tratta di un processo culturale condiviso in tutta Europa: "in the years 1945-1948 that were the moment not only of the division of Europe and the first stage of its postwar reconstruction but also, and in an intimately related manner, the period during which Europe's postwar memory was molded" (Judt 2000, 295-6). Quali operazioni sono essenziali per mettere in scena questa mancanza? In prima istanza, una grossolana dislocazione: le responsabilità di quanto avvenuto sono da attribuire interamente al nazionalsocialismo tedesco, secondo lo schema celebre individuato da Filippo Focardi, che oppone il cattivo tedesco al bravo italiano (Focardi 2013). Si tratta di un mito duraturo, tanto da essere cristallizzato nel titolo di un'opera tardiva di uno dei protagonisti del neorealismo, in uno degli esempi più significativi della stagione delle coproduzioni italo-sovietiche, Italiani brava gente (Giuseppe De Santis, 1964). Ma non mancano davvero i casi nella produzione dell'immediato dopoguerra, in cui la popolazione civile è pronta a offrire riparo a soldati alleati, come in Pian delle Stelle (Giorgio Ferroni, 1946) o finanche a fraternizzare con le due forze militari di occupazione, alleate e naziste, come in Vivere in pace (Luigi Zampa, 1946). Se il contributo nazionale alla Resistenza è spesso magnificato, la responsabilità di crimini e atrocità, associate alla devianza morale e sessuale, come in Roma, città aperta e Il sole sorge ancora, è attribuibile unicamente alle forze di occupazione tedesca. Questo spostamento o rifiuto di ogni responsabilità è la seconda operazione costituiva dell'atto mancato della narrazione traumatica del passato. La ripetizione di questo medesimo modello narrativo ha una funzione istruttiva e costruttiva: se, come suggerisce 


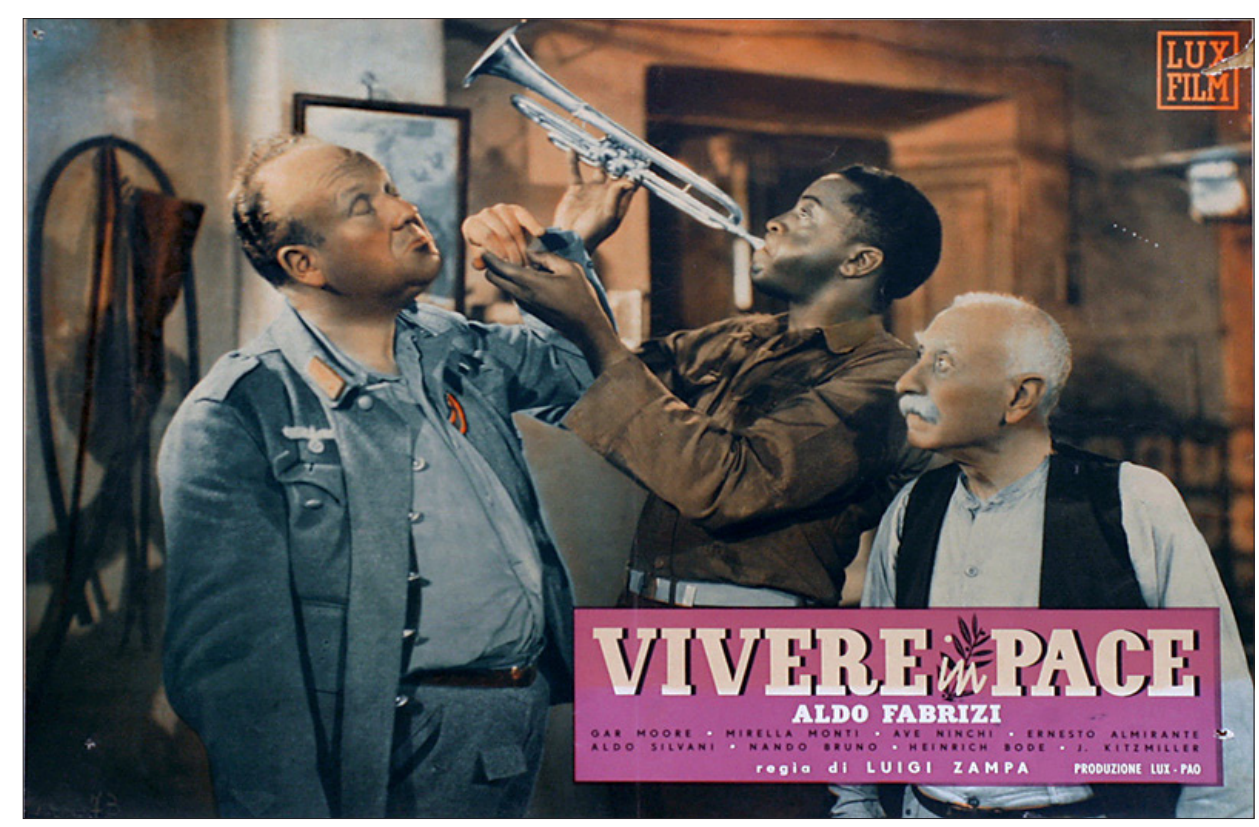

Fig. 3. "Vivere in pace" (L. Zampa, 1946). Il fraterno italiano.

Jedlowski, sulla scorta di Halbwachs, "la memoria collettiva non è infatti resurrezione o reviviscenza del passato in quanto tale. Essa è essenzialmente ricostruzione del passato in funzione del presente" (Jedlowski 1987, 21), allora la cultura e il cinema italiano del neorealismo hanno la funzione di costruire una memoria a corto raggio, celebrare una élite nazionale come epitome della nazione e sollevare quest'ultima da ogni responsabilità per accadimenti storici - totalitarismo, guerre coloniali e guerra d'aggressione - che, sul piano rappresentativo, sono del tutto assenti. L'iterazione di questo racconto agisce come una "memoria a bassa intensità", nei termini proposti dallo studioso tedesco Wulf Kansteiner: ad ampia circolazione, con un repertorio limitato di racconti, personaggi e immagini, ma con notevole efficacia nella modellazione di coordinate cognitive di base (Kansteiner 2002). Chi può negare che la morte di Pina (Anna Magnani) incinta, nel giorno del proprio matrimonio, falciata da una raffica di mitra tedesco per le vie di Roma, mentre cerca di raggiungere il proprio compagno rastrellato dalle truppe di occupazione in Roma, città aperta, non costituisca un'immagine emblematica di un racconto le cui coordinate di base vengono rinnovate a ogni variante? Una narrazione in cui la fertile e generosa nazione è 


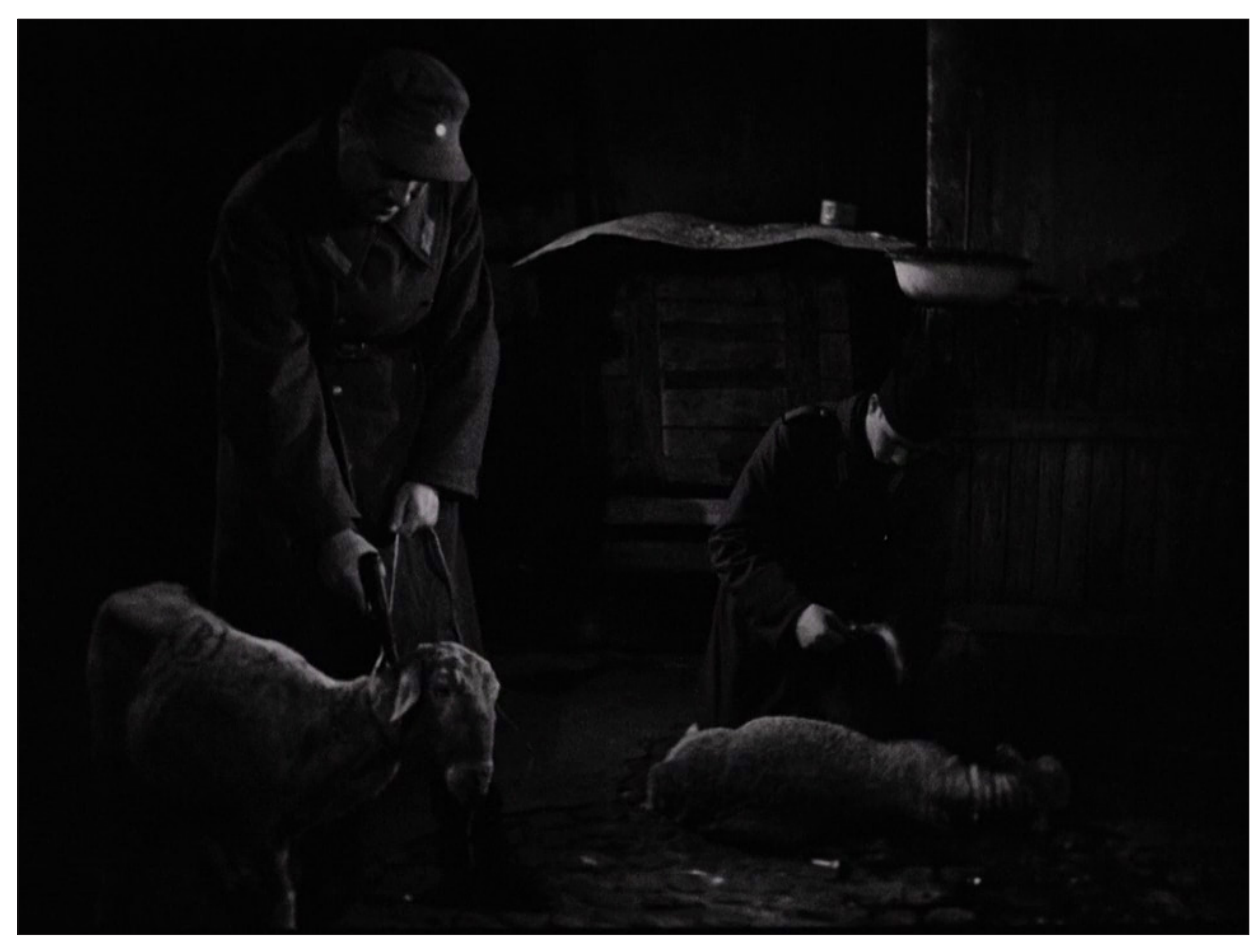

Fig. 4. "Roma, città aperta" (R. Rossellini, 1945): il sacrificio degli agnelli. L'italiano vittima inerme.

vulnerabile a una brutalità perversa, mentre la forza virile nazionale è indebolita? L'impotenza è perfettamente associabile alla vulnerabilità; entrambe costituiscono due condizioni necessarie di relative posizioni narrative e morali: vittima e testimone.

\section{Agnus dei. II sacrificio e lo sguardo}

Una delle sequenze meno spesso ricordate di Roma, città aperta offre una lettura allegorica del film. Forse, proprio la mobilitazione di un simile apparato iconografico e simbolico è la ragione della negligenza, data l'assunzione del film nel canone del realismo e della immediatezza, richiamata da Bazin. Due militanti del CLN, Francesco (Francesco Grandjacquet) e l'ing. Manfredi (Marcello Pagliero), si incontrano in una trattoria colla fidanzata di quest'ultimo, Marina (Maria Michi). Improvvisamente, fanno la propria apparizione dei soldati tedeschi che trascinano un agnello fin 
Fig. 5. "Irgendwo in Berlin" (G. Lamprecht, 1947). Dopoguerra europeo, vittimismo e rappresentazione dell'infanzia.

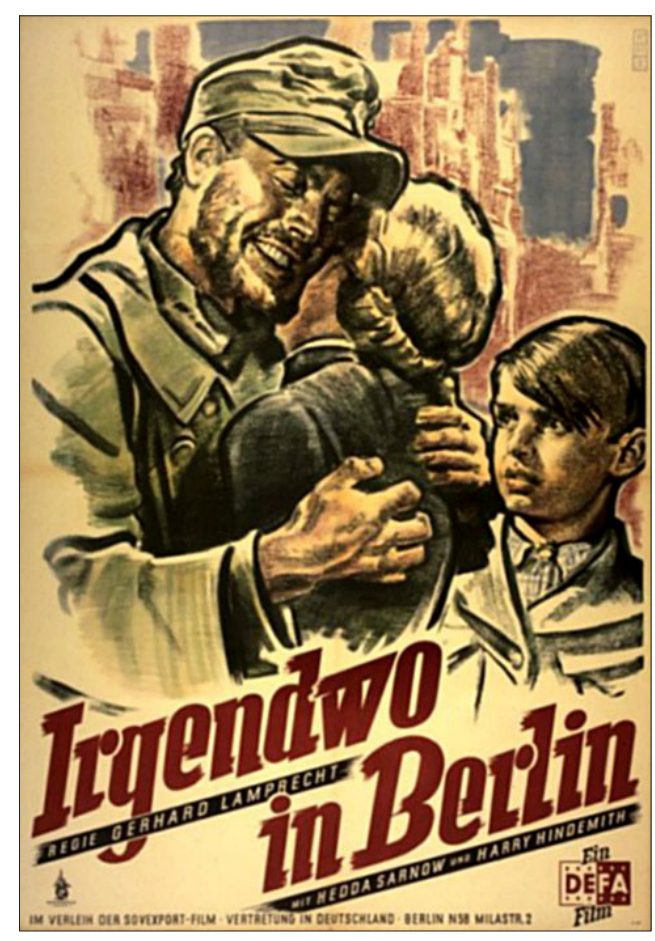

nel cortile retrostante il locale, dove lo uccidono con un'arma da fuoco, causando la reazione orripilata di Marina. Quello che si vuole qui ritenere è l'associazione della nazione con lo statuto di vittima sacrificale, alla stessa stregua in cui la simbologia dell'agnello di dio evoca l'olocausto dell'innocenza a beneficio dell'umanità intera. Se concordiamo con l'assunto di Aleida Assmann, secondo la quale "there are only three dignified roles for the national collective to assume, that of the victor who has overcome evil, that of the resistor who has heroically fought the evil and that of the victim who has passively suffered the evil" (Assman 2011, 54-5), la cultura italiana del secondo dopoguerra non poteva facilmente proporre il primo ruolo, con qualche impegno il secondo, ma con più disinvoltura il terzo: nel film, infatti, Manfredi, Francesco, Pina, Don Pietro (Aldo Fabrizi) e finanche Marina sono tutte vittime della abietta occupazione. Più vittime di tutti sono le figure dei bambini, sulla cui disperata sfilata, dopo aver assistito alla fucilazione del parroco e propria guida spirituale, si sofferma l'ultima inquadratura. Il dramma dell'infanzia è al centro di numerose realizzazioni europee, imperniate sulle vicende belliche o della difficile ricostruzione, dal tedesco Irgendwo in Berlin (Gerhard Lam- 
precht, 1947) alla produzione ungherese Valahol Európában (È accaduto in Europa, Géza Rádvanyi, 1947), dalla realizzazione austriaca Asphalt (Harald Röbbeling, 1951) alla coproduzione svizzero-statunitense The Search (Odissea tragica, Fred Zinnemann, 1948). Ma cosa rende la produzione cinematografica italiana tanto più significativa ed esemplare per la cultura cinematografica mondiale?

Si ritiene che il neorealismo cinematografico italiano abbia assunto tale rilevanza perché efficace nella costruzione di un modello narrativo ed enunciativo particolarmente congruente con la elaborazione di un "trauma culturale". Nella proposta teorica del sociologo statunitense Jeffrey C. Alexander, la condizione traumatica

is attributed to real or imagined phenomena, not because of their actual harmfulness or their objective abruptness, but because these phenomena are believed to have abruptly, and harmfully, affected collective identity. [...] For traumas to emerge at the level of collectivity, social crises must become cultural crises (Alexander 2004, 308).

Detto diversamente, il trauma culturale è il lavoro che un gruppo compie per ricostruire la propria identità, assegnando nuove funzioni a strutture significanti precedenti o creandone di nuove. Il cinema del neorealismo riconfigura in prima istanza le narrazioni eroiche del periodo interbellico, in cui i protagonisti virili e militareschi sono il vettore narrativo e morale di produzioni quali Ettore Fieramosca (Alessandro Blasetti, 1938), in cui Gino Cervi interpreta l'eponimo personaggio, Bengasi (Augusto Genina, 1941), in cui Fosco Giachetti veste i panni del capitano Berti, intento a resistere alle angherie degli inglesi, o Luciano Serra pilota (Goffredo Alessandrini, 1938), con Amedeo Nazzari che incarna un ardito aviatore, eroe di guerra nella Prima guerra mondiale e in Africa Orientale. Nel secondo dopoguerra questi divi sono oggetto di un'operazione di re-casting, con la quale mantengono i panni militari indossati fino a pochi anni prima, ma nelle vesti di reduci e sconfitti, spediti al fronte malgré eux e ora tornati in patria per confrontarsi con il disastro bellico, avvenuto senza che potessero opporvisi. Questo è il ruolo, rispettivamente, di Gino Cervi in Un uomo ritorna (Max Neufeld, 1946), Fosco Giachetti in La vita ricomincia (Mario Mattoli, 1945), o Amedeo Nazzari in Il bandito (Alberto Lattuada, 1946): riconfigurare gli eroi virili della narrazione cinematografica interbellica in vittime di decisioni che li sovrastano, la- 

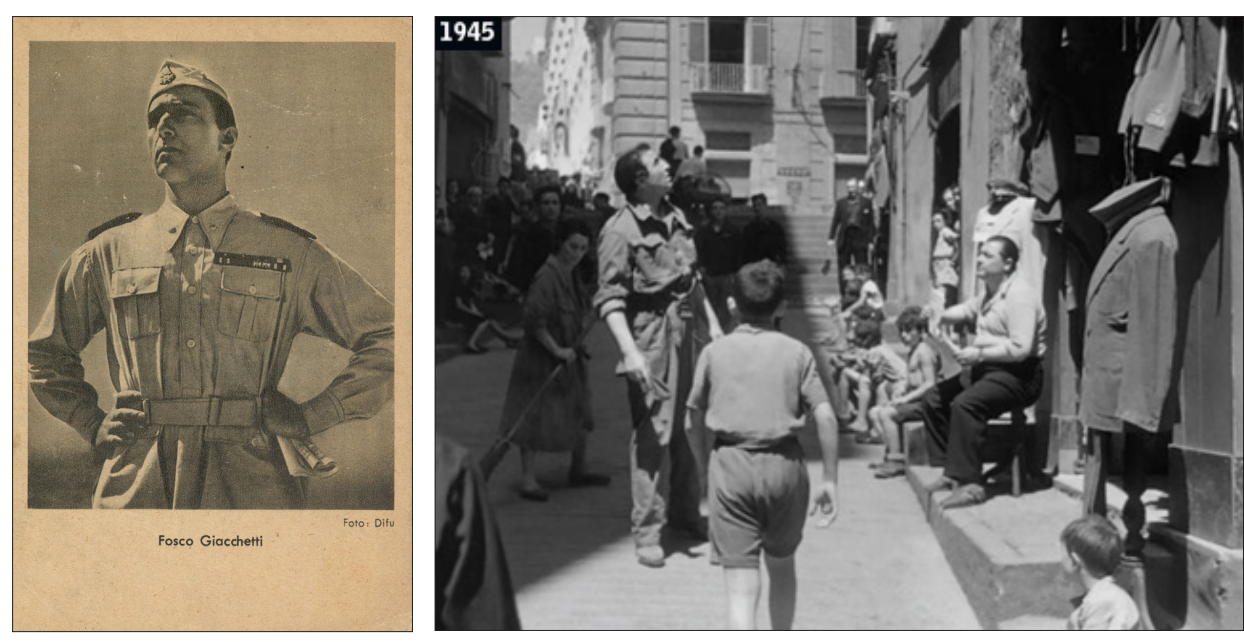

Figg. 6 e 7. Fosco Giachetti, da militare fascista in "Bengasi" (A. Genina, 1942) a reduce disorientato in "La vita ricomincia" (M. Mattoli, 1945). Riconfigurare la narrazione.

sciandoli alle prese con una devastazione dinanzi alla quale erano assenti, perché prigionieri. Preferibilmente in Germania. In questo senso, la dimensione traumatica, come sottolinea giustamente Elsaesser, travalica l'opposizione vittime/carnefici sul piano affettivo, dato che "in situations where everyone who has 'survived' or 'merely' comes after is prepared to embrace victim status” (Elsaesser 2014, 16). Ma si può ipotizzare un altro lavoro del trauma culturale proprio al cinema italiano del secondo dopoguerra, particolarmente capace di proiettarlo nell'arena internazionale: la creazione e promozione di una cultura mediatica umanitaria e compassionevole, fondata sulla esibizione dell'altrui sofferenza attraverso prodotti mediatici - segnatamente fotografia e cinema - per un pubblico internazionale, chiamato ad assistere alla sofferenza degli altri. Si tratta di quella che il sociologo francese Luc Boltanski definisce "politica della pietà" (Boltanski 2000). Fondata sulla distanza fisica di qualcuno più fortunato dalla sventura di qualcun altro, colmata attraverso la funzione dei media, la politica della pietà combina la esemplarità dei casi rappresentati - sono tutti esseri umani sofferenti - e la loro specificità, individualizzata e concretizzata dalle immagini fotografiche e cinematografiche. In questa prospettiva, il cinema del neorealismo rientra nel quadro di una nuova politica culturale internazionale, come forma di mediazione e conoscenza reciproca tra i popoli, che organismi quali la neonata UNESCO si propongono di promuovere. Come dichiarava nel 1951 Ross McLean, 


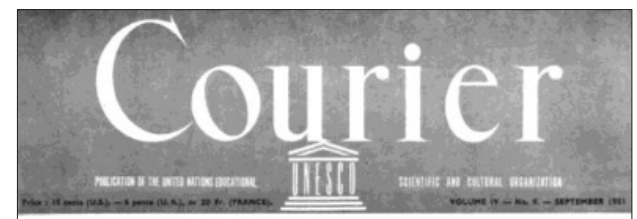

\section{SPECIAL ISSUE: THE CINEMA}

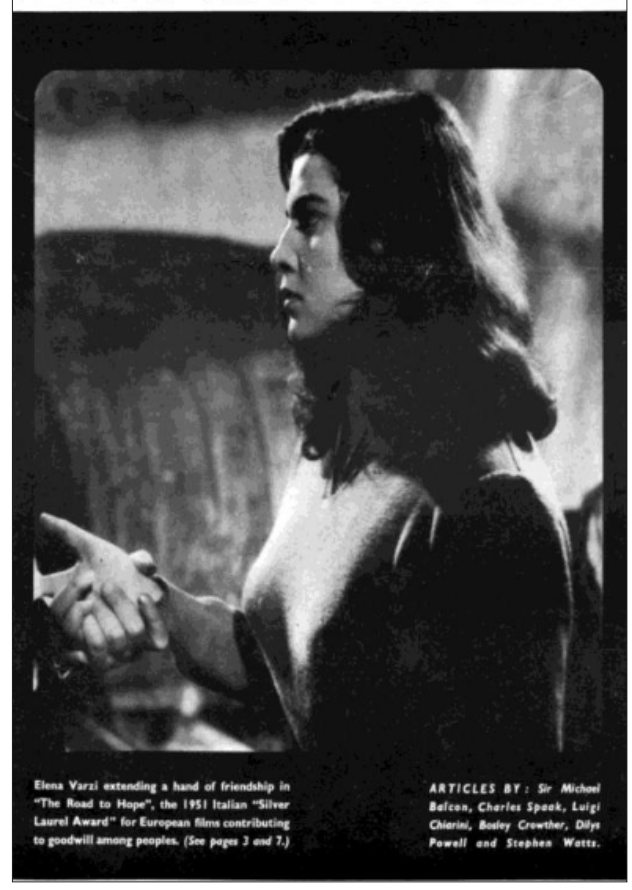

Fig. 8. Elena Varzi sulla copertina di "Courier" (September 1951). Nuove istituzioni sovranazionali, cultura visuale e politica della pietà.

a capo della ripartizione Films \& Visual Information, in un numero del bollettino UNESCO Courier dedicato al cinema e con un'immagine di Il cammino della speranza (Pietro Germi, 1950) in copertina: "The study of film as a means to human understanding is not a mere intellectual exercise. It is part of a continuing study we have all got to make in our search for harmony in a tortured world" (McLean 1951, 2). Le opere più importanti del neorealismo contribuiscono a questa "politica della pietà", secondo il teorico del cinema statunitense Karl Schoonover, grazie a un "umanesimo brutale" che itera un medesimo scenario: "an imperiled body is offered to a bystander's gaze as an opportunity to exercise moral judgement" (Schoonover 2012, xvi). Che sia la scena della tortura di Manfredi, cui Don Pietro è obbligato ad assistere in Roma, città aperta, il pestaggio del padre reo (Lamberto Maggiorani) visto dal figlio (Enzo 
Stajola) in Ladri di biciclette, l'annegamento di partigiani inermi dinanzi allo sguardo impotente dei G.I. americani in Paisà, o il suicidio conclusivo di Silvana (Silvana Mangano) sotto gli occhi di Francesca (Doris Dowling), in Riso amaro, la condizione di fragilità e vulnerabilità dell'essere umano è sempre offerta all'osservazione di un testimone incorporato nella diegesi. A questi il compito di trarre una considerazione etica e, al contempo, costruire una nuova estetica: il testimone. Questo osservatore ha un indubitabile vantaggio: è posto in una condizione di inazione per cause di forza maggiore e, pertanto, mai in quella di compiere azioni che possano introdurre una associazione tra una condotta e una morale; invece, la moralità risiede nel non prendere partito e, per riprendere l'espressione di Bazin, proporre un umanesimo radicale prima di ogni ideologia - essere (umani) prima di fare. Unitamente a esso, è un nuovo modello rappresentativo a imporsi, fondato meno sulla successione di azioni trasformative dell'universo diegetico e piuttosto sull'allentamento della concatenazione causale e sulla descrizione. Questa soluzione sembra essere, nel contesto culturale, mediatico e politico del secondo dopoguerra, in cui è necessario implementare un oblio dialogico, quella più opportuna per non fare i conti con il totalitarismo, con il collaborazionismo o anche solo con le lacerazioni del conflitto civile: amare la realtà di per se stessa, senza prendere partito e riflettere sul passato, è la soluzione ideale, a livello nazionale, per riannodare i legami sociali; a livello internazionale, per promuovere una cultura irenista e della comprensione reciproca, in cui il cinema gioca un ruolo fondamentale. Ma questa soluzione è il punto di convergenza ideale della molteplicità di esperienze maturate durante il Ventennio e negli anni della Seconda guerra mondiale? Questo umanesimo radicale sussume l'eterogeneità nazionale? A scostarsi appena dal corpus dei capolavori del cinema neorealista, alcune ombre si profilano.

\section{La linea bianca. II discorso documentario}

Uno dei film meno indagati della galassia neorealista è La città dolente (Mario Bonnard, 1948), sceneggiato tra gli altri da Federico Fellini, già collaboratore di Roberto Rossellini per le sceneggiature di Roma, città aperta e Paisà. I titoli di testa, subito dopo la denominazione del film propongono un "segmento preterizionale" (Bertoni 2007, 134-46), ovvero 


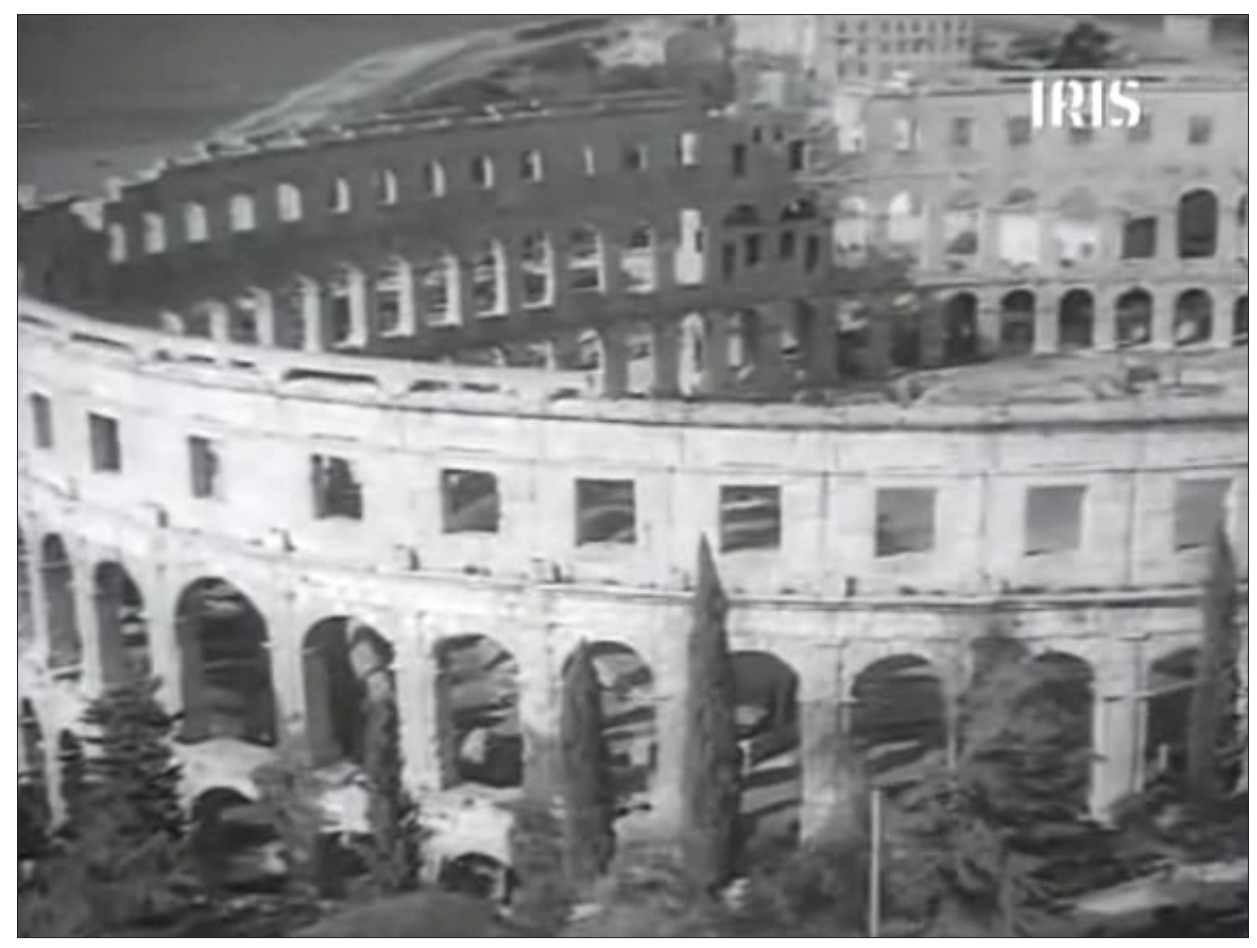

Fig. 9. La sequenza di apertura di "La città dolente" (M. Bonnard, 1948). Le memorie dell'Impero.

un inserto con cui si dichiara che quanto si vedrà è radicato nella realtà: "Da una storia vera un film", recita il cartiglio. Conclusi i titoli di testa, l'universo diegetico è costituito da un segmento documentario: una ampia panoramica sulla città di Pola, cui succedono immagini delle vestigia romane, della cristianità, del dominio della Serenissima, infine del Parco delle rimembranze. Vi fa seguito la documentazione visiva dei Trattati di Parigi del 1947, in virtù dei quali l'Italia cedeva alla Jugoslavia le province del Carnaro e Zara, l'alta valle dell'Isonzo e l'Istria, di cui faceva parte Pola; queste immagini sono sovrapposte a un manifesto, in cui si richiede l'autodeterminazione della popolazione polese a dispetto degli scenari internazionali. Conclude la sequenza l'esodo istriano: inquadrature della città svuotata e di rovine, infanzia e animali tra le masserizie, la popolazione in attesa di imbarcarsi sul piroscafo Toscana. A questa introduzione contribuisce una voce over, che descrive la presenza civilizzatrice italiana sin dalla romanità, ricorda i caduti della Prima guerra mondiale, rivendica il diritto della nazione sul territorio. Questa stessa voce over viene 
poi incarnata, con un'ardita soluzione enunciativa, nella soggettiva su un edificio da cui esce un attempato signore, pronto a partire sul Toscana: un controcampo rivela che la voce over è quella di un giornalista, che da presenza disincarnata e informativa si trasforma in personaggio fittizio. A questo incontro farà seguito la vicenda interamente finzionale di Berto, un operaio italiano persuaso da un amico militante comunista a non seguire il proprio popolo e la propria famiglia, per contribuire piuttosto all'edificazione della Jugoslavia: il nuovo regime perseguiterà entrambi, con ristrettezze crescenti, fino alla deportazione in campi di lavoro.

L'incipit del film dispiega soluzioni omologhe a quelle adottate dai capolavori del neorealismo: l'attestazione di verità di quanto andremo a vedere, nel cartiglio iniziale, come in La terra trema (Luchino Visconti, 1948) o Senza pietà (Alberto Lattuada, 1948); nell'impiego di immagini documentarie, come ulteriore strategia veridittiva della finzione conseguente, come in Paisà o Germania anno zero (Roberto Rossellini, 1948); e nella mobilità della voce over, che da "disembodied, [...] construed as fundamentally unrepresentable in human form, connoting a position of absolute mastery and knowledge outside the spatial and temporal boundaries of the social world the film depicts" (Wolfe 2016, 264), si incarna in un personaggiocerniera con la finzione successiva, come nell'incipit di Riso amaro. Eppure, il film tratta di un tema propriamente investito dal cono d'ombra dell'oblio dialogico: la questione del confine orientale e la controversa vicenda delle popolazioni italofone istro-giuliano-dalmate, della dominazione italiana sull'area nel periodo interbellico e del conflitto etnico, di classe e internazionale tra la fine del secondo conflitto mondiale e la definitiva reintegrazione della città di Trieste nel territorio italiano, nel 1954. Soprattutto, il segmento iniziale attraverso la concatenazione delle immagini e la voce over chiamata a commentarle propone una mappa del tempo rivelatrice di quanto negletto dai capolavori del neorealismo: una profondità storica che associa in un unico arco dominazione romana, veneziana e italiana, a promuovere una continuità nazionale fondata sull'opera civilizzatrice. Si tratta, evidentemente, di una memoria monologica, anziché dialogica, basata sul primato della nazione e della sua continuità su altre comunità di memoria, secondo una pratica largamente diffusa tra XIX e XX secolo, dato che, secondo Jeffrey K. Olick, "nations have had a special place in the history of memory and identity and in the history of their relations. Memory and the nation have a peculiar synergy" (Olick 2003, 2). 


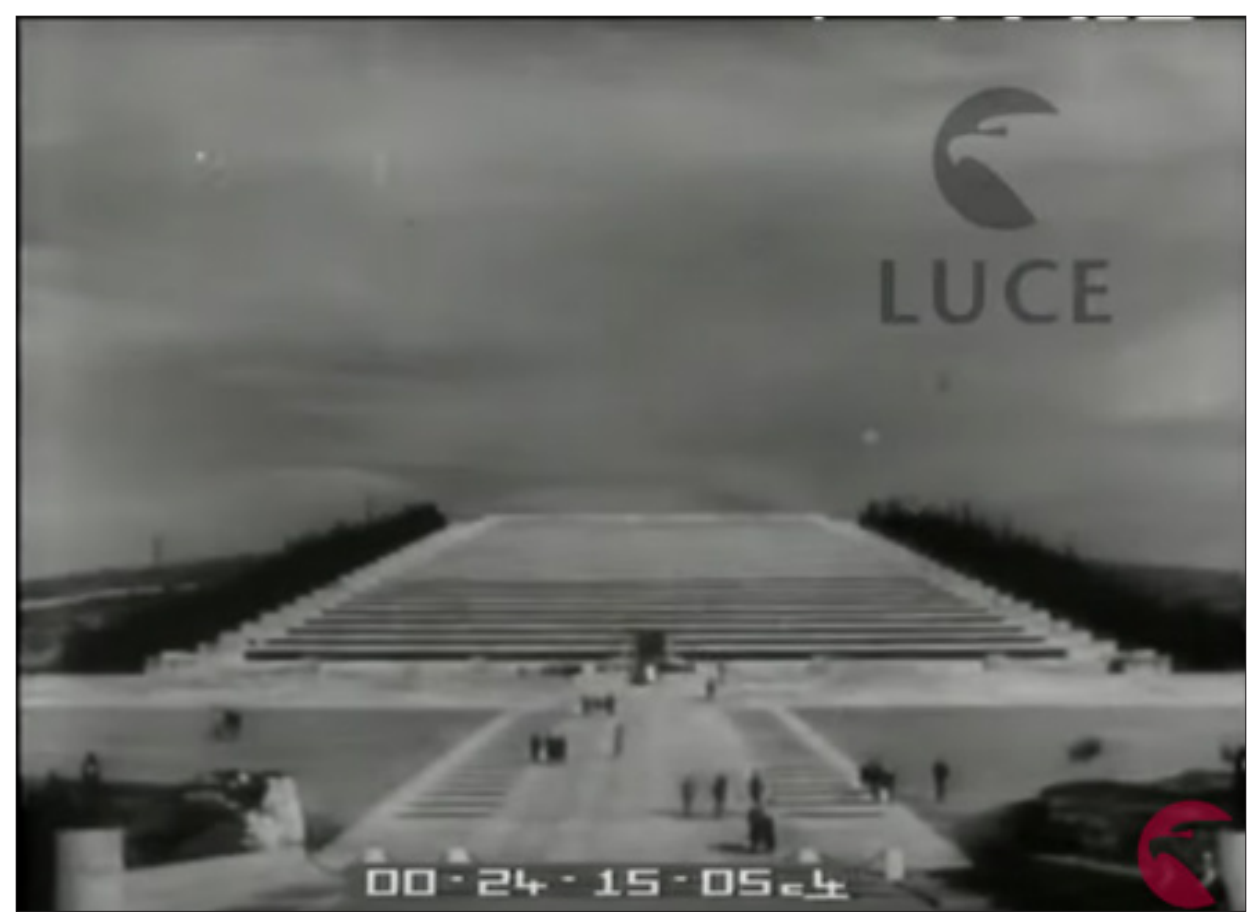

Fig. 10. "La Settimana Incom”, n. 32, "Da Trieste: cerimonia a Redipuglia” (14/11/1946). L'apparato simbolico irredentista.

A osservare la produzione documentaria e, in particolar modo, cinegiornalistica dedicata alla vicenda del territorio di Trieste emerge una prospettiva differente da quella proposta nei capolavori del neorealismo. O, meglio, a essa complementare, proprio nella esplicitazione di quanto l'immediatezza, l'amore per la realtà, l'umanesimo radicale e la densità fattuale delle immagini di quelli trascurano, ma che non cessa di funzionare come proprio atto mancato: il passato totalitario nazionale. Il corpus esaminato indaga due gruppi di film: quelli del fondo United States Information Service, preservati oggi all'Archivio Centrale dello Stato; e quelli di circolazione nazionale inclusi nei cinegiornali Notiziario Nuova Luce, La Settimana Incom e Mondo Libero, tra il 1945 e il 1954. L'amministrazione alleata della Zona A implicava infatti un sistema distributivo cinematografico differente dal resto del territorio italiano e un particolare impegno propagandistico da parte dell'USIS. È stato possibile ricostruire questo impegno grazie al ritrovamento, presso l'Archivio di Stato del capoluogo giuliano, di un fondo di pellicole dell'USIS (Barrera 
e Tosatti 2007; Crisanti 2014-15). Si è deciso di appuntare l'attenzione sulla modalità con cui la colonna visiva e quella sonora, centrale per i cinegiornali, definiscono lo spazio urbano, con particolare riguardo per le questioni dell'appartenenza nazionale e della memoria.

Possiamo partire da una considerazione complessiva. La colonna visiva di tutti i materiali visionati propone una definizione iconografica povera della città adriatica e del suo circondario. Sono sempre i medesimi luoghi, spesso raffigurati in modo simile o identico a essere proposti. Del tutto assenti le aree periferiche, invece ricorrenti nella rappresentazione dello spazio della Capitale e molto sporadica la rappresentazione del circondario della città. I luoghi individuati sono, in prevalenza, Piazza dell'Unità d'Italia' ${ }^{1}$, il Lungomare ${ }^{2}$, il porto e i cantieri navali ${ }^{3}$, la cattedrale di San Giusto ${ }^{4}$ e, più genericamente, il fervore della vita metropolitana. In una sola occorrenza compare il Borgo Teresiano ${ }^{5}$. Da questa rappresentazione emerge il quadro di una metropoli affacciata sul mare, identificata dal simbolo della riunificazione nazionale, sotto l'egida del cristianesimo e delle rovine della romanità. La mappa temporale disegnata di questi materiali documentari coincide con l'incipit di La città dolente. Non solo. Le immagini cinegiornalistiche e dei documentari

1 Per esempio, in La Settimana Incom 134, "Chiesto all'ONU: Trieste torni all'Italia" (25/3/1948); La Settimana Incom 142, "All'ombra di S. Giusto. Trieste attende" (10/4/1948); La Settimana Incom 242, "La giornata dell'esercito americano" (14/4/1949).

2 Esso ricorre in Notiziario Nuova Luce 12, "Meridiano d'Italia. Trieste: sfilata delle truppe alleate" (1946); La Settimana Incom 2, "Piccola posta. Vi risponde Silvana Jachino" (22/2/1946); La Settimana Incom 276, "La giornata dell'esercito americano” (14/4/1949); La Settimana Incom 482, "Trieste. Inaugurata la Fiera internazionale” (30/8/1950).

3 Si vedano i cinegiornali La Settimana Incom 445, "Varo della motonave "Australia" (24/5/1950); La Settimana Incom 447, "Sbarcata la $4^{\circ}$ milionesima tonnellata" (26/5/1950); La Settimana Incom 476, "Trieste. Varo della motonave 'Oceania" (9/5/1950); La Settimana Incom 499, “Trieste. Varo della motonave 'Neptunia” (6/10/1950) e il documentario Trieste industriale (Tullio Mainardi, s.i.d.).

4 Per esempio, Vita triestina 2 (Tullio Mainardi, s.i.d.), Notiziario Nuova Luce 12, "Meridiano d'Italia. Trieste: sfilata delle truppe alleate" (1946); La Settimana Incom 8, "A Trieste" (10/4/1946); La Settimana Incom 134, "Chiesto all'ONU: Trieste torni all'Italia” (25/3/1948); La Settimana Incom 242, “La giornata dell'esercito americano” (14/4/1949).

5 La Settimana Incom 2, "Piccola posta. Vi risponde Silvana Jachino" (22/2/1946). 
realizzati per l'USIS da Tullio Mainardi, operatore del Circolo Cinematografico Triestino, spesso riattivano la memoria dell'irredentismo nazionale attraverso la mobilitazione dell'apparato simbolico presente sul territorio cittadino e nei suoi dintorni. Per esempio, il Faro della Vittoria, costruito tra 1923 e 1927 per commemorare i marinai italiani caduti durante il primo conflitto mondiale; il Monumento ai Caduti, edificato nel 1935 sul Colle di San Giusto e il Parco delle Rimembranze ${ }^{\gamma}$; il sacrario di Redipuglias. Un armamentario monumentale e ideologico approntato durante il Ventennio viene ricuperato per celebrare l'identità italiana della città e rivendicarne l'appartenenza in una fase contrastata. Questa operazione si celebra in associazione con la glorificazione della ricostruzione: se la colonna visiva dei film non offre mai immagini di distruzione del tessuto urbano, e solo in un'occasione vengono mostrati i relitti di navi nel porto cittadino, le immagini spesso descrivono il modernismo di nuovi edifici e il varo di transatlantici, raffigurati come esito conclusivo di una piena riattivazione di una realtà industriale e titanica nelle proprie strutture. Questa medesima modernità urbana è promossa dai servizi dedicati alle fiere campionarie ${ }^{10}$.

In che modo illustrare la condizione peculiare del territorio triestino? La più parte dei materiali visionati non descrive mai il contesto frontaliero. Quando ciò avviene, la sintassi oppone un circondario pattugliato da oscure truppe difficilmente individuabili, intente a sorvegliare armate il territorio e a vessare una popolazione femminile inerme ${ }^{11}$. Viceversa, lo spazio cittadino e, segnatamente, il Lungomare sono il luogo in cui sfi-

6 Per esempio, La Settimana Incom 450, “Trieste. Inaugurata la nuova sede dell'AGIS" (1/6/1950).

7 La Settimana Incom 815, "Il prefetto Vitelli a Trieste" (24/7/1952); La Settimana Incom 989, "Il problema di Trieste" (10/9/1953).

8 La Settimana Incom 32, "Da Trieste: cerimonia a Redipuglia” (14/11/1946).

9 La Settimana Incom 445, "Varo della motonave "Australia" (24/5/1950); La Settimana Incom 476, "Trieste. Varo della motonave 'Oceania" (9/5/1950); La Settimana Incom 499, "Trieste. Varo della motonave 'Neptunia”" (6/10/1950); La Settimana Incom 520, "Varo della motonave 'Augustus' a Trieste" (24/11/1950).

10 Per esempio, Vita triestina (Tullio Mainardi, s.i.d.); La Settimana Incom 482, "Trieste. Inaugurata la Fiera internazionale (30/8/1950).

11 La Settimana Incom 142, "All'ombra di S. Giusto. Trieste attende" (10/4/1948). 

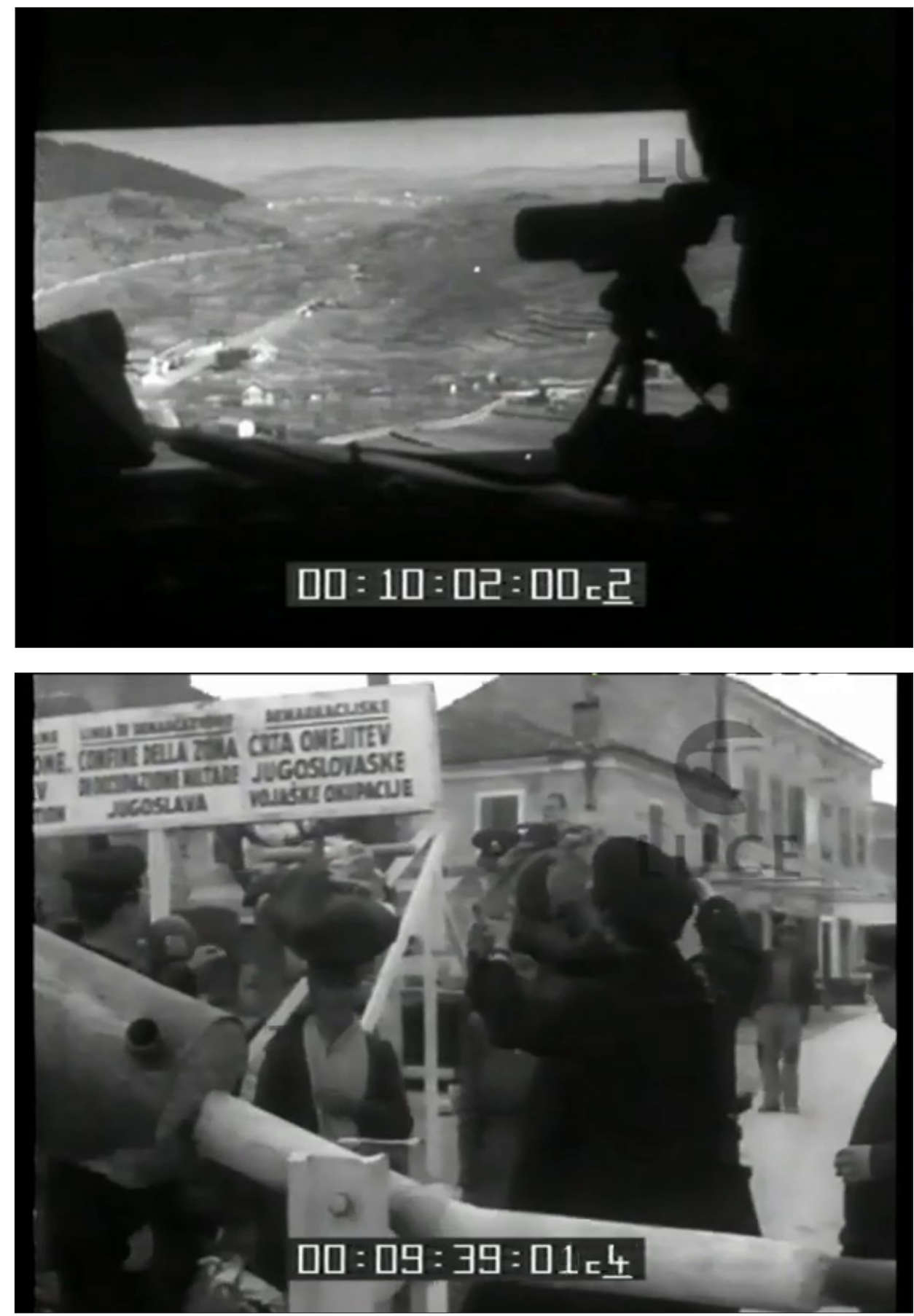

Figg. 11-12. "La Settimana Incom”, n. 142, “All'ombra di S. Giusto. Trieste attende” (10/4/1948). L'oscura minaccia sull'italianità inerme. 
lano luminose le forze alleate; almeno fino agli scontri del marzo 1952. Questa identità cittadina, priva di un esterno e illuminata dalla presenza degli Alleati, è rafforzata dai frequenti richiami allo European Recovery Plan e da una modernizzazione che concerne anche l'antropologia urbana: in un servizio della Settimana Incom, Trieste è sede del primo corpo di polizia femminile, in qualche modo omologo a quanto accade di là dall'Atlantico ${ }^{12}$.

La sintassi dispiegata da questi cinegiornali compie tre operazioni principali. Associa le masse popolari con la propria dirigenza, raffigurata in prima istanza dal sindaco democristiano Gianni Bartoli, e da ulteriori cariche pubbliche (prefetti, rettori), secondo un modulo consueto dei cinegiornali, imperniati sulle occasioni ufficiali. Connette la città con il resto del territorio nazionale, che a vario titolo rivendica a sé Trieste attraverso commemorazioni e dimostrazioni. Collega le vicende cittadine con i grandi tavoli delle trattative internazionali, in cui i protagonisti della politica mondiale (occidentale) discutono del futuro del territorio triestino. Sul piano della colonna sonora, possiamo individuare due tratti distintivi. La presenza soverchiante della voce over e l'impiego in funzione ideologica della colonna sonora extradiegetica. La voce over ricorrentemente richiama l'indiscutibile identità e appartenenza italiana di Trieste, il sacrificio sofferto per la sua integrazione nazionale durante la Prima guerra mondiale e l'importanza dell'esperienza irredentista; occasionalmente, nel caso di Mondo Libero, che adotta uno stile discorsivo più disinvolto di La Settimana Incom, si giunge all'impiego della prima persona singolare e del dialetto triestino per comporre un'ode a Trieste, in occasione delle dimostrazioni del marzo $1952^{13}$. La musica extradiegetica fa ampio ricorso a motivi musicali identificativi la nazione tutta (Fratelli d'Italia) ${ }^{14}$, di richiamo patriotico (Va' pensiero $)^{15}$, o di chiara associazione con le vicende della Prima guerra mondiale, grazie all'uso frequente di La canzone del Piave' ${ }^{16}$. Trieste, nel

12 La Settimana Incom 532, "La polizia femminile a Trieste" (22/12/1950).

13 Mondo Libero 13, "Trieste mia” (27/3/1952).

14 La Settimana Incom 7, "La grande manifestazione d'italianità" (1/4/1946).

15 La Settimana Incom 302, "Trieste ha votato" (15/6/1949).

16 Essa si individua in La Settimana Incom 191, "Associazioni triestine e istriane sfilano per le strade della Capitale" (23/9/1948); Mondo Libero 20, "Passione di Trieste”(5/4/1952). 
commento sonoro, è soggetta a una minaccia oscura, mai identificata, che fa il paio con le forze militari che cingono il centro urbano, senza definirne l'appartenenza.

Che operazione compie nella costruzione della memoria collettiva questo corpus di film, esaminati come un insieme relativamente omogeneo per coerenza produttiva e discorsiva? Proviamo a trarre un rapido bilancio. La memoria richiede iterazione: "Later access or recall of memories is greatly enhanced by the retelling of these narratives, either by individuals alone or in public" (Winter e Sivan 1999, 14). I cinegiornali, in prima istanza, grazie ai due dispositivi di legge n. 379 (1947) e n. 958 (1949), nella dieta mediatica nazionale sono una presenza costante che itera determinati schemi narrativi e una specifica iconografia. Grazie all'impiego di una serie limitata di luoghi e iconemi e di motivi sonori ripetuti, questi materiali compiono esattamente un'operazione rituale di ricostruzione della memoria, a partire dall'iterazione dei medesimi temi. Inoltre, sul piano iconografico questo corpus riattiva l'iconografia irredentista realizzata dal fascismo per avocare il territorio triestino alla nazione, mentre disegna una mappa temporale che dalla romanità alla cristianità passa per la Prima guerra mondiale per giungere alla modernizzazione contemporanea. Un elemento viene espunto dalla narrazione: il periodo mussoliniano, in toto. Ne restano tuttavia le tracce proprio nella iconografia convocata. L'esibizione di una mancanza, in qualche misura. Inoltre, sul piano sonoro, la continuità storica di Trieste è tracciata tra Risorgimento, Prima guerra mondiale e fase postbellica. In secondo luogo, sul piano degli spazi pubblici, i luoghi identificativi della città e del territorio sono quelli emblematici della memoria o, in alternativa, quelli della modernità tecnologica e industriale: sedi di un racconto in cui il progresso nazionale conquista le proprie tappe più ambiziose, eventualmente grazie all'ausilio dello European Recovery Plan. Da ultimo, questo territorio ha, nelle immagini delle occasioni pubbliche e nel commento sonoro, uno statuto incerto: circondato da una minaccia oscura, esso è connesso tuttavia alla madrepatria e allo scenario internazionale e, nella metafora più volte ripresa, è un ponte per l'Europa. Questo corpus ci chiarisce in qualche misura il lavoro del trauma culturale nella transizione italiana dal totalitarismo alla democrazia e della nazione dalla condizione di aggressore belligerante a quella di vittima. Infatti, documentari e cinegiornali mantengono una prospettiva storica in cui la 
comunità nazionale è protagonista di una progressione ineluttabile dai fasti della Roma imperiale alle conquiste della modernità: questa avanzata lineare espunge dal proprio racconto il Ventennio mussoliniano. Se i capolavori del neorealismo semplicemente muovono dalla fine del Fascismo, documentari e cinegiornali lo saltano a piè pari, rivelandone tuttavia la corposa assenza. Come ben sintetizza Giacomo Lichtner, "The neorealist orthodoxy was the cinematic reflection of a peace based not on the solidity of awareness and justice but on the inclusiveness of selective memory and amnesty" (Lichtner 2013). In secondo luogo, se, come rammenta John Foot, della Resistenza non vennero creati monumenti commemorativi nazionali (Foot 2009, 147-52), non altrettanto si può dire dell'esperienza della Prima guerra mondiale e dello sforzo durante il Ventennio di commemorarla con un apparato monumentale di considerevole impegno e dimensioni, tra cui il caso del sacrario di Redipuglia (1938) è uno dei più rilevanti. Ora, è questo apparato a essere mobilitato per costruire memoria e identità nazionali, laddove per il territorio triestino quest'ultima e la stessa appartenenza alla comunità statuale italiana viene messa in discussione. Il che equivale a dire che il discorso documentario seguitava a far riferimento a una cultura visiva e simbolica elaborata durante il Ventennio, che informava la memoria nazionale in termini non propriamente dialogici. Da ultimo, questa memoria collettiva si fonda, in buona sostanza, sul monologo: le forze oscure che minacciano Trieste, epitome della nazione, non sono mai esplicitamente nominate e possono far riferimento tanto al nazismo quanto al socialismo. Se i capolavori del neorealismo promuovono la condizione di testimone insieme a quella di vittima, quest'ultima prevale nei materiali documentari, laddove l'identità e il territorio nazionale siano nuovamente messi a repentaglio. Di più. Nei cinegiornali essa è associata a quella del soggetto vittorioso della latinità o dell'irredentismo.

La questione della responsabilità collettiva e individuale del totalitarismo, della guerra di aggressione o della tensione interetnica nelle aree del confine orientale è esclusa dal discorso delle immagini. Il lavoro del trauma culturale, necessario a rifondare il legame sociale, poteva riassegnare nuove collocazioni a vecchi significati ed eliminare i più problematici di essi. Ma, accanto alle nuove strade tracciate dalle soluzioni più originali ed efficaci per la diplomazia culturale, restavano i vecchi territori e le mappe più consunte per attraversarli. 


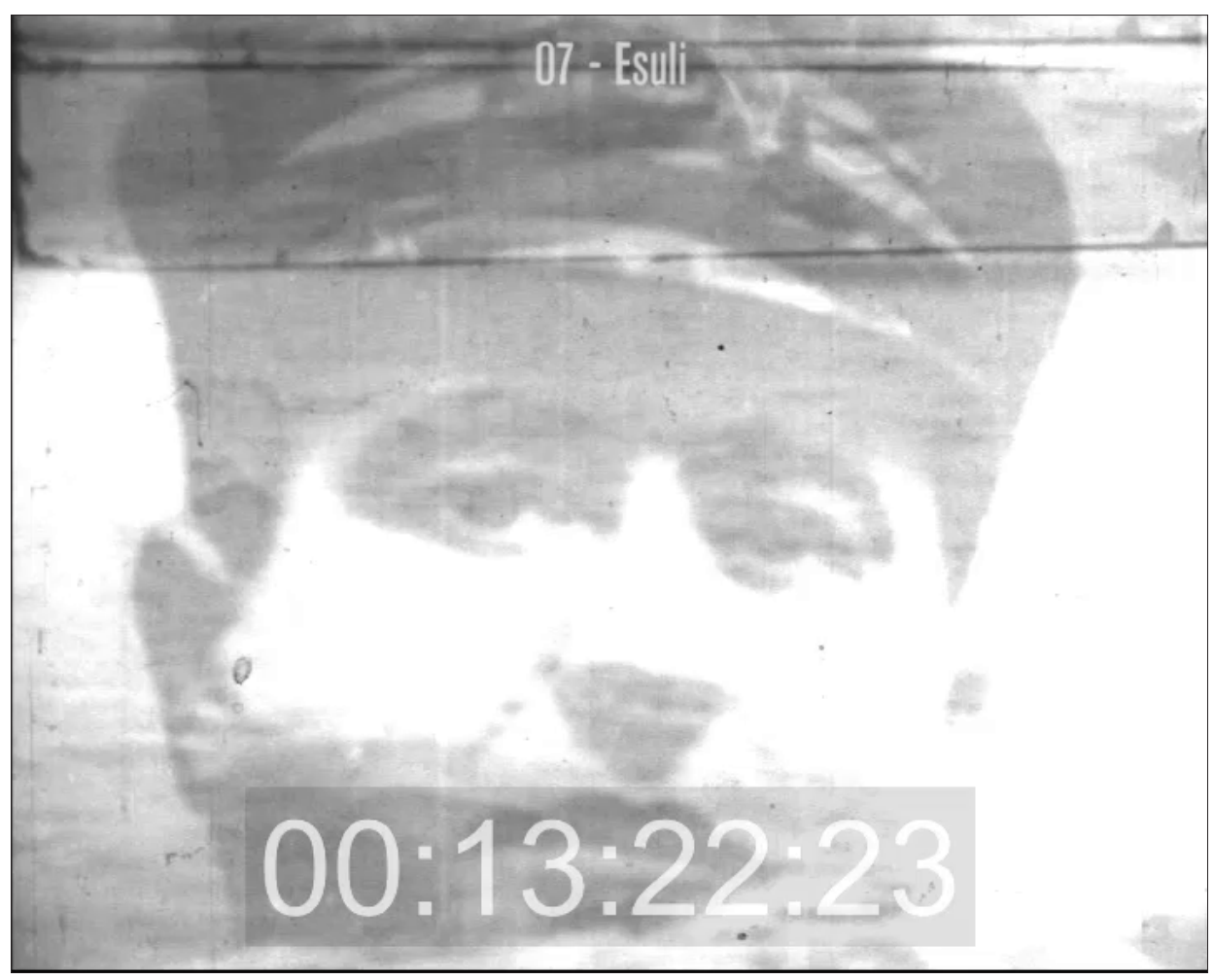

Fig. 13. Nazario Sauro sulle onde dell'Adriatico in "Esuli" (T. Mainardi, 1948). La memoria dell'irredentismo.

\section{Bibliografia}

Adorno, Theodor Wiesengrund. 1972. "Critica della cultura e società." In Prismi. Saggi sulla critica della cultura, di Theodor Wiesengrund Adorno, 3-21. Torino: Einaudi.

Alexander, Jeffrey C. 2004. "Toward a Theory of Cultural Trauma." In Cultural Trauma and Collective Identity, a cura di Jeffrey C. Alexander, Ron Eyerman, Bernard Giesen, Neil Smelser e Piotr Sztompka, 1-30. Berkeley: University of California Press.

Assmann, Aleida. 2011. "From Collective Violence to a Common Future: Four Models for Dealing with a Traumatic Past." In Cultural History and Literary Imagination, a cura di Martin Modlinger e Philipp Sonntag, 44-62. Oxford: Peter Lang.

Barrera, Giulia e Giovanna Tosatti, a cura di. 2007. United States Information Service di Trieste. Catalogo del fondo cinematografico (1941-1966). Roma: Ministero per i Beni e le Attività Culturali. Direzione Generale per gli Archivi.

Bazin, André. 1986. "Il realismo cinematografico e la scuola italiana della Liberazione." In Che cosa è il cinema?, di André Bazin, 275-303. Milano: Garzanti.

Bertoni, Federico. 2007. Realismo e letteratura. Una storia possibile. Torino: Einaudi. 
Boltanski, Luc. 2000. Lo spettacolo del dolore. Morale umanitaria, media e politica. Milano: Raffaello Cortina.

Brunetta, Gian Piero. 1996. "La ricerca dell'identità nel cinema italiano del dopoguerra." In Identità italiana e identità europea nel cinema italiano, a cura di Gian Piero Brunetta, 11-67. Torino: Fondazione Giovanni Agnelli.

Crisanti, Giulia. 2014-15. "Le politiche d'informazione americane in Italia e il Fondo USIS di Trieste (1941-1966).” Tesi di Laurea Magistrale, Università degli Studi di Pisa. https://etd.adm.unipi.it/t/etd-06112015-085119/ (ultima cons. 18 ottobre 2020).

Elsaesser, Thomas. 2014. German Cinema - Terror and Trauma. Cultural Memory since 1945. London-New York: Routledge.

Focardi, Filippo. 2013. Il cattivo tedesco e il bravo italiano. Roma-Bari: Laterza.

Foot, John. 2009. Italy's Divided Memory. Basingstoke: Palgrave Macmillan.

Halbwachs, Maurice. 1987. La memoria collettiva. Milano: Unicopli.

- 1997. I quadri sociali della memoria. Santa Maria Capua Vetere: Ipermedium.

Jedlowski, Paolo. 1987. "Introduzione." In La memoria collettiva, di Maurice Halbwachs, 7-33. Milano: Unicopli.

Judt, Tony. 2000. "The Past Is Another Country: Myth and Memory in Postwar Europe." In The Politics of Retribution in Europe. World War II and Its Aftermath, a cura di István Deák, Jan T. Gross e Tony Judt, 293-323. Princeton: Princeton University Press.

Kansteiner, Wulf. 2002. "Finding Meaning in Memory: A Methodological Critique of Collective Memory Studies." History and Theory 41 (2): 179-197.

Leavitt, Charles. 2016. “'An Entirely New Land'? Italy's Post-war Culture and Its Fascist Past." Journal of Italian Modern Studies 21 (4): 4-18.

Lichtner, Giacomo. 2013. Fascism in Italian Cinema Since 1945. The Politics and Aesthetics of Memory. Basingstoke: Palgrave Macmillan.

McLean, Ross. 1951. "International Understanding and the Cinema." Courier 4 (8): 2.

Minghelli, Giuliana. 2012. Landscape and Memory in Post-Fascist Italian Film. Cinema Year Zero. London-New York: Routledge.

Olick, Jeffrey K. 2003. "Introduction." In States of Memory. Continuities, Conflicts, and Transformations in National Retrospection, a cura di Jeffrey K. Olick, 2-16. Durham: Duke University Press.

Olick, Jeffrey K., Vered Vinitzky-Seroussi e Daniel Levy. 2011. "Introduction.” In The Collective Memory Reader, a cura di Jeffrey K. Olick, Vered Vinitzky-Seroussi e Daniel Levy, 4-62. Oxford-New York: Oxford University Press.

Schoonover, Karl. 2012. Brutal Vision. The Neorealist Body in Postwar Italian Cinema. Minneapolis: University of Minnesota Press.

Wagstaff, Christopher. 2007. Italian Neorealist Cinema. An Aesthetic Approach. Toronto: University of Toronto Press. 
Winter, Jay ed Emmanuel Sivan. 1999. "Setting the Framework." In War and Remembrance in the Twentieth Century, a cura di Jay Winter ed Emmanuel Sivan, 6-39. Cambridge: Cambridge University Press.

Wolfe, Charles. 2016. "Historicizing the 'Voice of God': The Place of Voice Over Commentary in Classic Documentary." In The Documentary Film Reader. History, Theory, Practice, a cura di Jonathan Kahana, 264-80. Oxford: Oxford University Press.

Zavattini, Cesare. 2002. "L'Associazione Culturale Cinematografica Italiana." In Opere. Cinema, a cura di Valentina Fortichiari e Mino Argentieri, 662-6. Milano: Bompiani.

Zerubavel, Eviatar. 2005. Mappe del tempo. Memoria collettiva e costruzione sociale del passato. Bologna: Il Mulino. 
